University of Wollongong

Research Online

Faculty of Engineering and Information

Faculty of Engineering and Information

Sciences - Papers: Part B

Sciences

2018

\title{
Thin Silicon Microdosimeter utilizing 3D MEMS Fabrication Technology: Charge Collection Study and its application in mixed radiation fields
}

\author{
Linh T. Tran \\ University of Wollongong, tltran@uow.edu.au \\ Lachlan Chartier \\ University of Wollongong, Ic752@uowmail.edu.au \\ Dale A. Prokopovich \\ ANSTO, dalep@uow.edu.au \\ David Bolst \\ University of Wollongong, dbolst@uow.edu.au \\ Marco Povoli \\ SINTEF
}

See next page for additional authors

Follow this and additional works at: https://ro.uow.edu.au/eispapers1

Part of the Engineering Commons, and the Science and Technology Studies Commons

Research Online is the open access institutional repository for the University of Wollongong. For further information contact the UOW Library: research-pubs@uow.edu.au 


\title{
Thin Silicon Microdosimeter utilizing 3D MEMS Fabrication Technology: Charge Collection Study and its application in mixed radiation fields
}

\author{
Abstract \\ New $10-\mu$ m-thick silicon microdosimeters utilizing 3-D technology have been developed and investigated \\ in this paper. The TCAD simulations were carried out to understand the electrical properties of the \\ microdosimeters' design. A charge collection study of the devices was performed using $5.5-\mathrm{MeV} \mathrm{He}^{2+}$ \\ ions which were raster scanned over the surface of the detectors and the charge collection median \\ energy maps were obtained and the detection yield was also evaluated. The devices were tested in a 290 \\ $\mathrm{MeV} / \mathrm{u}$ carbon ion beam at the Heavy Ion Medical Accelerator in Chiba (HIMAC) in Japan. Based on the \\ microdosimetric measurements, the quality factor and dose equivalent out of field were obtained in a \\ mixed radiation field mimicking the radiation environment for spacecraft in deep space. \\ Disciplines \\ Engineering | Science and Technology Studies \\ Publication Details \\ Tran, L. T., Chartier, L., Prokopovich, D. A., Bolst, D., Povoli, M., Summanwar, A., Kok, A., Pogossov, A., \\ Petasecca, M., Guatelli, S., Reinhard, M. I., Lerch, M., Nancarrow, M., Matsufuji, N., Jackson, M. \& \\ Rosenfeld, A. B. (2018). Thin Silicon Microdosimeter utilizing 3D MEMS Fabrication Technology: Charge \\ Collection Study and its application in mixed radiation fields. IEEE Transactions on Nuclear Science, 65 \\ (1), 467-472.

\section{Authors} \\ Linh T. Tran, Lachlan Chartier, Dale A. Prokopovich, David Bolst, Marco Povoli, Anand Summanwar, Angela \\ Kok, Alex Pogossov, Marco Petasecca, Susanna Guatelli, Mark I. Reinhard, Michael L. F Lerch, Mltchell \\ John Bromley Nancarrow, Naruhiro Matsufuji, Michael A. Jackson, and Anatoly B. Rosenfeld
}




\section{Thin Silicon Microdosimeter utilizing 3D MEMS Fabrication Technology: Charge Collection Study and its application in mixed radiation fields}

Linh T. Tran, Lachlan Chartier, Dale A. Prokopovich, David Bolst, Marco Povoli, Anand Summanwar, Angela Kok, Alex Pogossov, Marco Petasecca, Susanna Guatelli, Mark I. Reinhard, Michael Lerch, Mitchell Nancarrow, Naruhiro Matsufuji, Michael Jackson and Anatoly B. Rosenfeld

\begin{abstract}
New 10- $\mu$ m-thick silicon microdosimeters utilizing 3D technology have been developed and investigated in this work. The TCAD simulations were carried out to understand the electrical properties of the microdosimeters' design. A charge collection study of the devices was performed using a $5.5 \mathrm{MeV}$ $\mathrm{He}^{2+}$ ions which were raster scanned over the surface of the detectors and the charge collection median energy maps were obtained and the detection yield was also evaluated. The devices were tested in a $290 \mathrm{MeV} / \mathrm{u}$ carbon ion beam at the heavy ion medical accelerator in Chiba (HIMAC) in Japan. Based on the microdosimetric measurements, the quality factor and dose equivalent out-of-field were obtained in a mixed radiation field mimicking the radiation environment for spacecraft in deep space.
\end{abstract}

Index Terms-Microdosimetry, heavy ion therapy, silicon microdosimeter, 3D mesa, charge collection, IBIC, dose

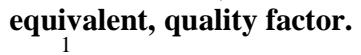

\section{INTRODUCTION}

$\mathrm{M}$ ixed radiation fields are those composed of radiation of different types such as ions, electrons, gamma and neutrons of different energies. The monitoring of ionizing radiation and determination of dose equivalent in mixed radiation fields, such as: those in high energy medical and research accelerators, commercial and military flights at high altitude and in spacecraft. The outer space environment represents a major challenge due to difficulties caused by the

\footnotetext{
${ }^{1}$ L. T. Tran, L. Chartier, D. Bolst, Alex Pogossov, M. Petasecca, M. L. F. Lerch and A. B. Rosenfeld are with the Centre for Medical Radiation Physics, University of Wollongong, NSW 2522, Australia (e-mail: tltran@uow.edu.au; lc752@uowmail.edu.au; db001@uowmail.edu.au; apogosso@tpg.com.au; marcop@uow.edu.au, mlerch@uow.edu.au; anatoly@uow.edu.au).

D. A. Prokopovich and M. I. Reinhard are with the NSTLI Nuclear Stewardship Platform, Australian Nuclear Science and Technology Organisation (ANSTO), Lucas Heights, NSW 2234, Australia (e-mail: dale.prokopovich@ansto.gov.au; mark.reinhard@ansto.gov.au).

M. Povoli, A. Summanwar and A. Kok are with SINTEF, Norway. (email: marco.povoli@sintef.no; angela.kok@sintef.no).

N. Matsufuji is with Research Centre for Charge Particle Therapy, National Institute of Radiological Science, Japan. (email: matsufuji.naruhiro@qst.go.jp).

M. Jackson is with the University of New South Wales, NSW 2052, Australia. (email: Michael.Jackson2@health.nsw.gov.au )

M. Nancarrow is with Electron Microscopy Centre, University of Wollongong, Wollongong, New South Wales 2500, Australia. (email: nancarro@uow.edu.au)
}

necessity to properly identify the contribution to the dose equivalent of each radiation component. The International Commission on Radiation Units and Measurements (ICRU) report 40 introduced the quality factor, $\mathrm{Q}$, to weight the absorbed dose for biological effectiveness of the charged particles producing the absorbed dose [1]. Dosimetry of mixed fields for radiation protection purposes (dose equivalent determination) requires extremely complex dosimetric systems consisting of specific spectroscopy detectors for each radiation component. Another method to determine the dose equivalent in mixed radiation fields is microdosimetry. Regional microdosimetry is based on the measurement of ionizing energy deposited in a micron sized volume of similar dimensions to biological cells. The energy deposition in such a volume is called the lineal energy deposition $(y): \mathrm{y}=\frac{E}{\langle l\rangle}$, where $E$ is the energy deposited in a micron sized sensitive volume (SV) with a mean chord length $\langle l\rangle$ and the spectrum of stochastic events $f(y)$ for all primary and secondary charged particles. With knowledge of the microdosimetric spectra of a radiation field $\left(y^{2} f(y)\right.$ vs $\left.\log (y)\right)$, the dose equivalent can be derived using:

$$
\mathrm{H}=D \int Q(y) y^{2} f(y) d(\log (y))
$$

where $Q(y)$ is a Quality Factor obtained from radiobiological experiments and $D$ is the absorbed dose [1].

The gold-standard detector used for microdosimetry is the tissue equivalent proportional counter (TEPC) which is advantageous due to its spherical SV and tissue equivalency through use of a tissue-equivalent gas and walls. However, TEPCs have several limitations such as high voltage operation requirements up to thousands of volts, large and bulky size of assembly, which reduces its spatial resolution, as well as wall effects [2] and an inability to simulate multiple cells.

The Centre for Medical Radiation Physics (CMRP) has developed a number of generations of microdosimeters on silicon-on-insulator (SOI) substrates which have been successfully tested [3-8] and recently summarized in [9]. The latest development of SOI microdosimeters at CMRP is the 3D microdosimeter using 3D MEMS technology at SINTEF MiNaLab, Norway.

3D detector technology was introduced in 1995 by S. Parker for high-energy physics applications. Many advantages of the 3D detector have been proven including radiation hardness, rapid charge collection, and low depletion voltage. 
The developments of micro-machining and standard VLSI (Very Large Scale Integration) technologies, together with advanced deep reactive ion etching (DRIE) with high depth-towidth ratios make 3D detector fabrication possible [10]. The CMRP in collaboration with SINTEF have applied patented technology and have fabricated fully 3D SV array microdosimeters (also called "mushroom" microdosimeters). The feasibility studies have been performed earlier with different types of 3D detectors for microdosimetry applications $[11,12]$. This paper presents two significant structures of 3D mushroom microdosimeters, their electrical and charge collection properties and preliminary results of dose equivalent obtained with these devices in a mixed radiation field.

\section{MATERIAL AND METHOD}

\section{A. Test structure of 3D "mushroom" microdosimeters}

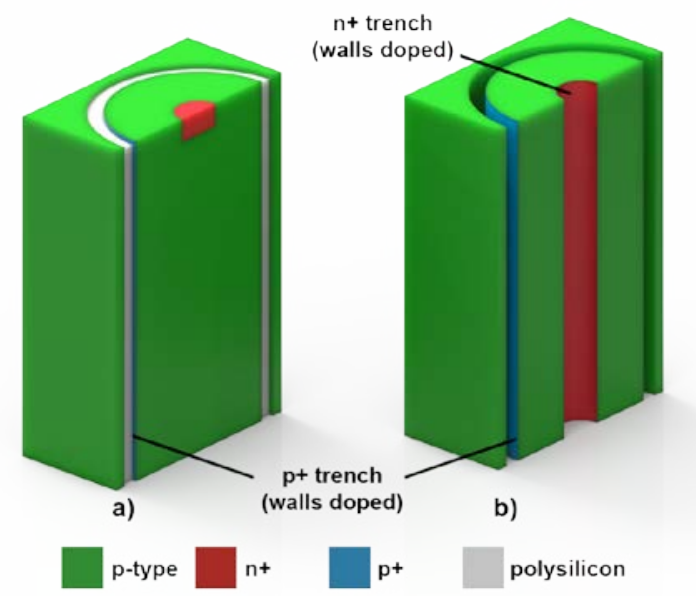

Fig. 1 Simplified schematics illustrating different configurations designed to define sensitive volume geometry and charge collection properties. a) Trenched planar structure and b) Trenched 3D structure.

Fig 1 shows the schematic of single 3D SVs of two different structures. The first mushroom microdosimeter structure on the right is called a trenched 3D and consists of 3D cylindrical SVs with a core column of air and $\mathrm{n}^{+}$doping in the inner core walls of the SV center (Fig.1b). Each SV is surrounded with a trench of air with $\mathrm{p}^{+}$doping on the outer wall, designed to physically eliminate the possibility of charge generated outside the SV from being collected. In this structure, the $\mathrm{p}^{+}$trench and $\mathrm{n}^{+}$column of the SV is not filled with polysilicon, therefore the trench cannot be a closed cylinder. In order to electrically connect SVs in an array, two half-moon trenches were made leaving some silicon to allow passage for the metal contacting the inner $\mathrm{n}^{+}$electrode. Outer $\mathrm{Al}$ buses were connected to $\mathrm{p}^{+}$ outer electrodes of 3D SVs. Scanning Electron Microscope (SEM) images clearly showing the structure of the fabricated microdosimeter are shown in Fig. $2 \mathrm{~b}$.

The second structure of the mushroom microdosimeter is called a trenched planar, which also consists of 3D cylindrical SVs with a planar $\mathrm{n}^{+}$core produced by ion implantation (planar technology). Each SV is surrounded with a complete $\mathrm{p}^{+}$ doped trench filled with polysilicon (Fig 1a). SEM images of this device's structure show that a complete trench was etched (Fig. 3a) and the trench was filled with polysilicon (showed in Fig. 3b).

Both structures are based on an array of 3D cylindrical SVs with diameters of $18 \mu \mathrm{m}$ or $30 \mu \mathrm{m}$, fabricated on a high resistivity p-SOI with a $10 \mu \mathrm{m}$ thick active layer bonded to a low resistivity supporting wafer and $2 \mu \mathrm{m}$ silicon oxides in between. The SVs are separated into odd and even arrays (Fig. 2a). A P-stop layer has been deposited everywhere on both device structures and under the pad to avoid metal-oxidesemiconductor (MOS) build up charge effect under the metal buses.

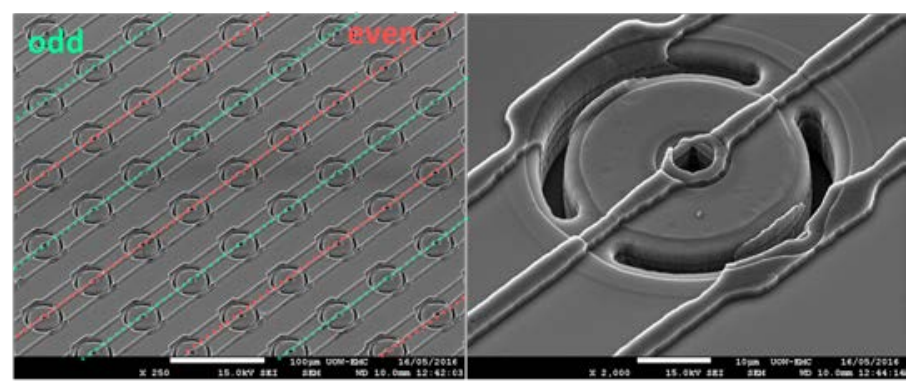

a)

b)

Fig. 2 SEM images of trench 3D mushroom microdosimeter with air filled $\mathrm{n}^{+}$column and $\mathrm{p}^{+}$trench filled with air. a) array of SVs and b) single SV view.

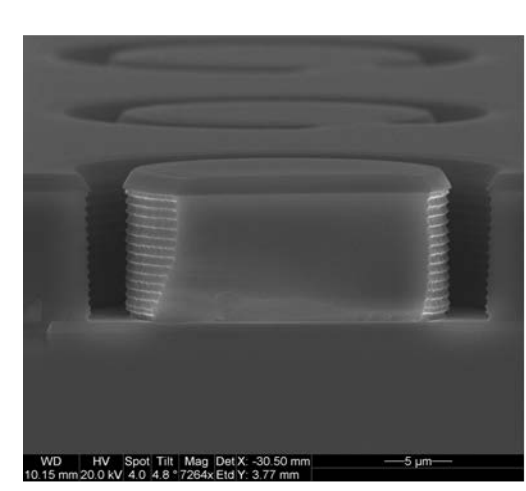

a)

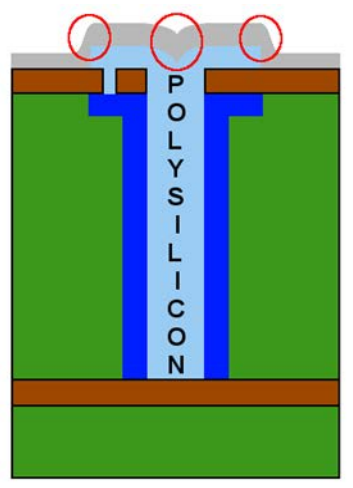

b)
Fig. 3 a) SEM image of a trench planar mushroom microdoismeter with a complete trench, b) trench filled with polysilicon $\mathrm{p}+$ column

\section{B. TCAD modeling}

SYNOPSYS TCAD tools were used to optimize the device's design and to simulate the detector's performance. Modeling of the electric field distribution of trenched 3D single SV was performed. The geometry of the SOI microdosimeter used in this simulation is shown in Fig 4. A bias voltage of $70 \mathrm{~V}$ was applied to the microdosimeter model.

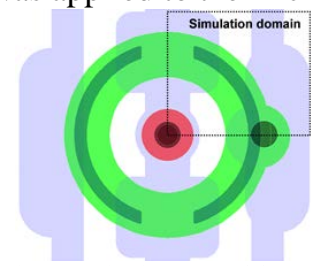

Fig. 4 A full layout of the trenched 3D sensitive volume filled with air in TCAD simulations. Simulation domain is on the top right of the image 


\section{Charge Collection Study}

The charge collection efficiency for the mushroom microdosimeters was investigated using the ion induced charge collection (IBICC) technique with the Heavy Ion Microprobe at ANSTO [13]. The IBICC measurements utilized a microbeam of $5.5 \mathrm{MeV} \mathrm{He}^{2+}$ ions focused to a diameter of approximately $1 \mu \mathrm{m}$ which were raster scanned over the surface of the sample. The amount of energy deposited in the microdosimeter was measured using an AMPTEK A250 charge sensitive preamplifier and Canberra 2025 Shaping Amplifier with $1 \mu$ s shaping time. The signal was fed directly to a Canberra 8701 analog to digital converter (ADC) of the data acquisition system. The signal corresponding to the beam's $X$ and $Y$ position as well as the charge collection $E$ for each event was processed into an event-by-event list mode file. The data was processed into median charge collection image maps for spatial correlation of the energy deposition of the scanned area. The energy calibration was performed using a calibrated pulse generator which was calibrated with a $300 \mu \mathrm{m}$ thick planar silicon fully depleted PIN diode with $100 \%$ Charge Collection Efficiency (CCE) in response to $5.5 \mathrm{MeV}$ ${ }^{2+}$ He ions.

\section{Determination of dose equivalent in mixed radiation field}

The 3D mushroom microdosimeters were irradiated at the Heavy Ion Medical Accelerator in Chiba (HIMAC), located at the National Institute of Radiological Science (NIRS) in Japan. The facility consists of ion sources, a radio frequency quadrupole linear accelerator (LINAC) for low speed ions and an Alvarez LINAC for medium speed ions as an injector to the two synchrotrons, with maximum energy of $400 \mathrm{MeV} / \mathrm{u}$ [14].

A $60 \mathrm{~mm}$ spread out Bragg peak (SOBP) produced with $290 \mathrm{MeV} / \mathrm{u}{ }^{12} \mathrm{C}$ ions was used in this experiment. Mixed radiation produced out-of-field from primary ${ }^{12} \mathrm{C}$ ion interactions including fragments, neutrons and gamma to some extent represents the exposure scenario mission into deep space environment. This work aims at presenting method of dose equivalent determination in unknown mixed radiation field.

The 3D mushroom microdosimeter was connected to a low noise spectroscopy-based readout circuit, allowing lineal energy measurements as low as $0.15 \mathrm{keV} / \mu \mathrm{m}$. The probe was then placed in a water tank and measured at different depths in water. The dose equivalent was calculated for positions outside of the primary radiation field including the distal edge of the SOBP.

The dose equivalent $(H)$ is given by:

$$
H=D_{T E} \bar{Q}=D_{T E} \int Q(y) d(y) d y
$$

where $Q(y)$ is a Quality Factor obtained from ICRU 40 [1], $D_{T E}$ is the absorbed dose in tissue and $d(y)$ is dose lineal energy distribution. $Q(y)$ is given by:

$$
Q(y)=\frac{a_{1}}{y}\left[1-e^{\left(-a_{2} y^{2}-a_{3} y^{3}\right)}\right]
$$

With coefficients:

$$
\begin{aligned}
\mathrm{a}_{1} & =5510 \mathrm{keV} / \mu \mathrm{m} \\
\mathrm{a}_{2} & =5.10^{-5} \mu \mathrm{m}^{2} / \mathrm{keV}^{2}
\end{aligned}
$$

$$
\mathrm{a}_{3}=2.10^{-7} \mu \mathrm{m}^{3} / \mathrm{keV}^{3}
$$

The absorbed dose to silicon is calculated by integrating the MCA energy deposition spectra in silicon SVs and dividing by the mass of the silicon 3D mushroom SVs. The $D_{T E}$ can then be obtained by multiplying the dose to silicon by the average tissue equivalent conversion factor of 0.58 . This method was used previously in our work [15].

\section{RESULTS}

\section{A. TCAD modeling}

Fig. 5 shows a result of purely electrical simulations. The SV was biased using a voltage ramp applied to the $\mathrm{p}^{+}$trench and the distribution of the electric field was observed. The electric field profiles on the right side are extracted along the radius of the cell at 3 different depths (front surface, middle of the device and back surface). Close to the front side, a large electric field peak was found, it was due to the $\mathrm{n}^{+}$doping forming a junction with the p-spray isolation layer. This was expected and the final layout accounts for an over-hanging metal able to split the primary electric field peak in two smaller ones, increasing the maximum operating voltage of the device.

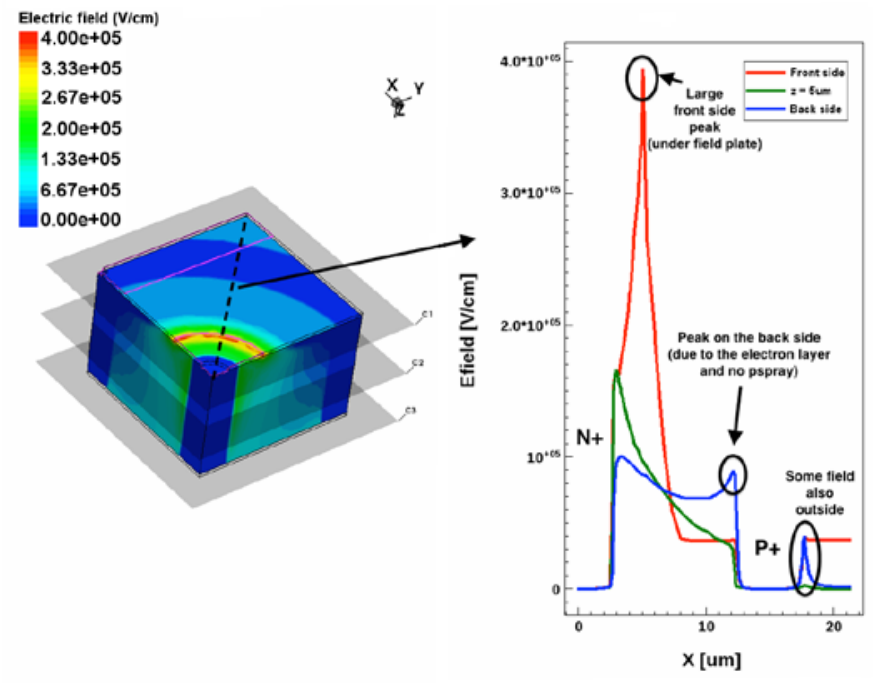

Fig. 5 Simulated electric field distribution for a sensitive volume of radius $12.5 \mu \mathrm{m}$. The $3 \mathrm{D}$ simulation results are shown on the left. On the right are $1 \mathrm{D}$ cuts extracted from the structure radially at 3 different depths into the sensitive volume. The applied bias voltage was $70 \mathrm{~V}$

Another charge collection simulation was performed for a 3D trenched sensitive volume where the $\mathrm{p}^{+}$trench is not filled with polysilicon. Some silicon was left to allow passage for the metal contacting the inner $\mathrm{n}+$ electrode. In this simulation, the effect of the opening in the trench on timing properties of 3D SV was investigated. The layout and a cross-section of the simulated cell along with the results for a bias voltage of $6 \mathrm{~V}$ are shown in Fig. 6. This investigation was conducted using a Minimum Ionizing Particle (MIP), releasing $80 \mathrm{e}^{-} / \mu \mathrm{m}$ into the silicon bulk, and hitting the device in 3 different locations: inside the intended active area, just outside of the trench opening and far outside of the trench opening. The obtained current pulses as a function of time show extremely fast charge collection inside the sensitive area. At the same time, it was 
found that, even for particles hitting the device outside of the trench delimiting the active volume, some signal could still be extracted, although lower in magnitude and much slower in time evolution. This happens because the depletion region can exit the opening in the trench and, since lifetimes in high resistivity silicon are long, charge carriers have enough time to diffuse and reach the depleted region and can be collected. Given that the signals generated outside the cell are much smaller in magnitude and slower in time, these effects can be mitigated with the use of a threshold on the readout system and/or using faster shaping times.

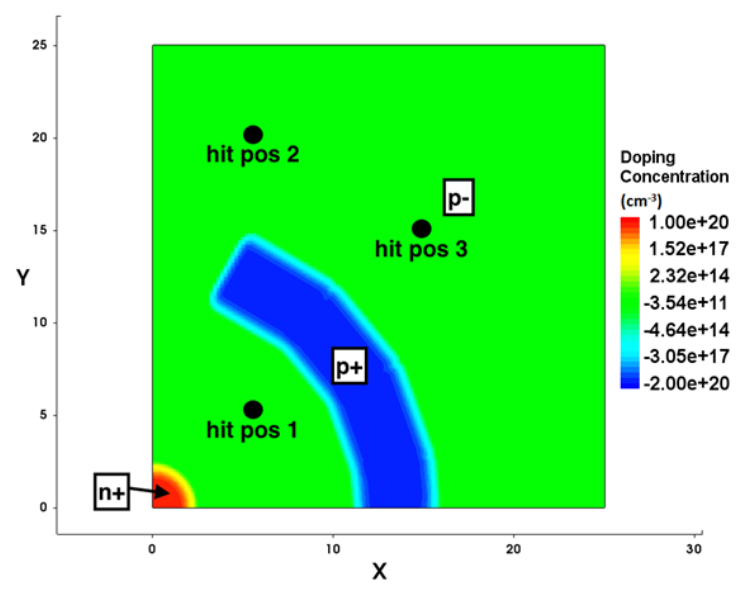

a)

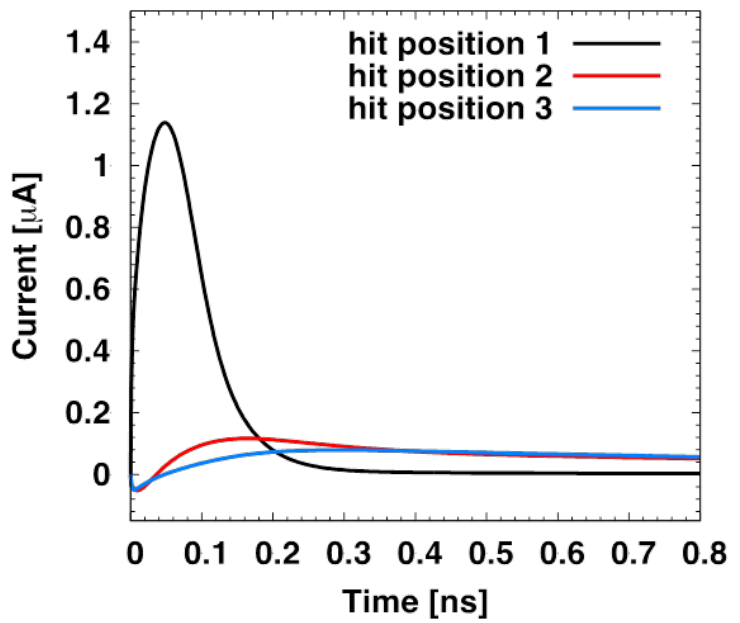

b)

Fig. 6 Simulation results for a sensitive volume with empty trenches (air), for different minimum ionizing particles hit positions. A slice of the 3D simulated structure with the corresponding particle hit positions is shown in (a) and the resulting current pulses versus time are shown in (b) for an applied bias voltage of $6 \mathrm{~V}$.

\section{B. Response of the 3D mushroom microdosimeters to $5.5 \mathrm{MeV}$ $\mathrm{He}^{2+}$ ions microbeam}

The 3D mushroom microdosimeters were scanned with a $5.5 \mathrm{MeV} \mathrm{He}^{2+}$ ion microbeam at different locations. Fig. 7a and $7 \mathrm{~b}$ show the multichannel analyzer (MCA) spectra and the median energy map respectively obtained by the trenched 3D microdosimeter. The peak of the deposited energy distribution in the 3D detector is approximately $1260 \mathrm{keV}$ which is slightly less than the expected energy deposition of $1367 \mathrm{keV}$ from 5.5
$\mathrm{MeV} \mathrm{He}^{2+}$ ion in $9.1 \mu \mathrm{m}$ thick silicon device covered by 0.85 $\mu \mathrm{m} \mathrm{SiO}_{2}$ overlayer on the front of the detector, calculated using the Geant4 simulation toolkit. The thickness values of silicon layer and oxide overlayer were taken from SEM crosssection images. This result shows that the charge collection efficiency of this device structure is over 92\%. The events in the high energy peak between 1100 and $1400 \mathrm{keV}$ are coming from within the sensitive volume, as expected. There is a void where no charge collection occurs in the middle of the SV.

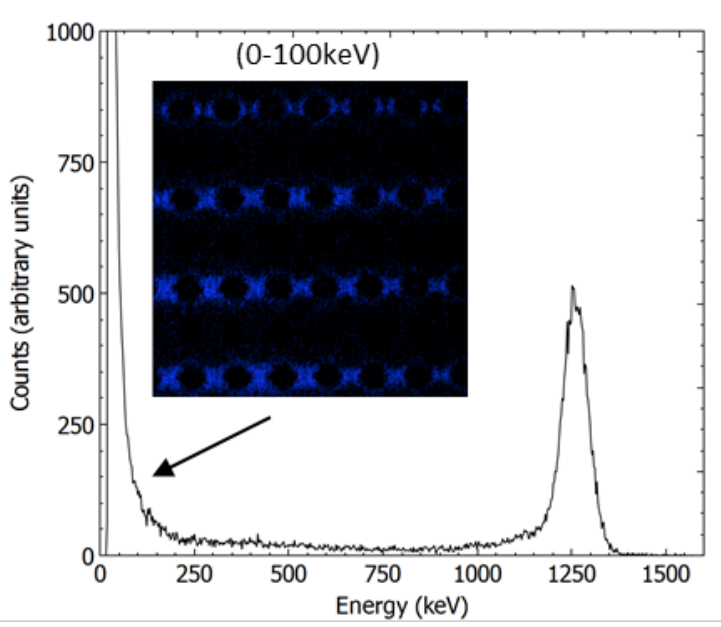

a)

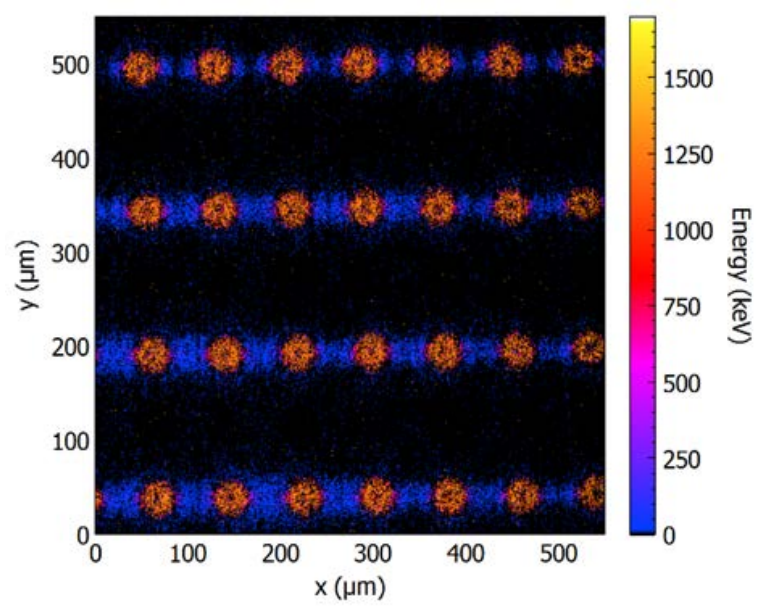

b)

Fig. 7 Response of even array of the trenched 3D mushroom microdosimeter to $5.5 \mathrm{MeV} \mathrm{He}^{2+}$ a) Energy spectrum and b) median energy map for $10 \mathrm{~V}$ bias. Odd array were grounded.

The low energy events from $0-100 \mathrm{keV}$ are seen to be primarily from the silicon bridges that connect each sensitive volume, and some events occurring around the sensitive volume. Shown in Fig 7a is the MCA spectrum obtained with the median energy map for energy window $0-100 \mathrm{keV}$ corresponding to the bridge region between the SVs.

Fig. 8a and 8b show the MCA spectra and the median energy maps obtained by the trenched planar microdosimeter in response to $5.5 \mathrm{MeV} \mathrm{He}^{2+}$ ion beam, respectively. The peak of the deposited energy distribution in the trenched planar microdosimeter is approximately $1305 \mathrm{keV}$ which is slightly higher than the trenched 3D microdosimeter, demonstrating 
slightly better charge collection efficiency (96\%) for the planar $\mathrm{n}^{+}$core structure. Fig 8a also presents the median energy maps for different energy windows corresponding to the MCA spectrum at the same scan. The low energy events from 0-50 $\mathrm{keV}$ occur outside the SV, even outside of the polysilicon trench. This could be from charge diffused through the polysilicon into the SV drift region. It can be seen that the charge collection within the SVs is quite uniform confirming that the design with planar $\mathrm{n}^{+}$core is suitable (Fig. 8b). The energy window of $1100-1200 \mathrm{keV}$ in the MCA spectrum is corresponding to the deficit of collected charge by $\mathrm{He}^{2+}$ ions in the planar cores.

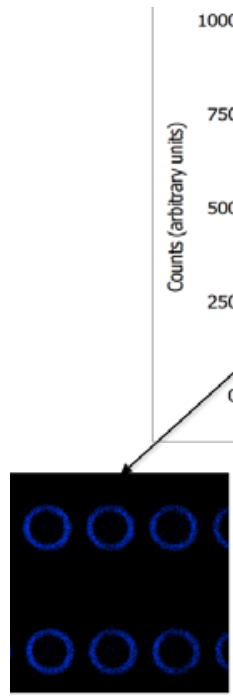

$0-50 \mathrm{keV}$

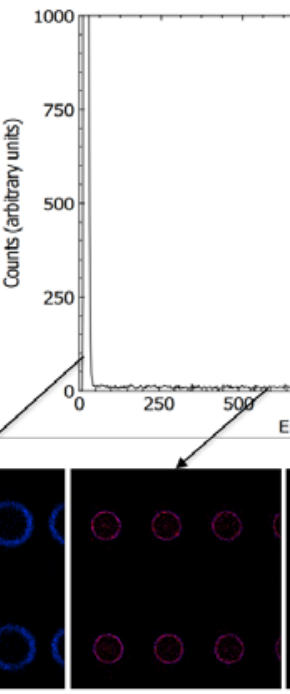

$50-1100 \mathrm{keV}$

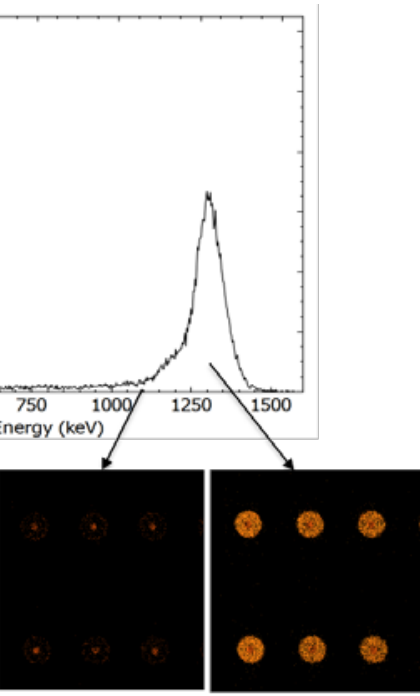

$1100-1200 \mathrm{keV}$

a)

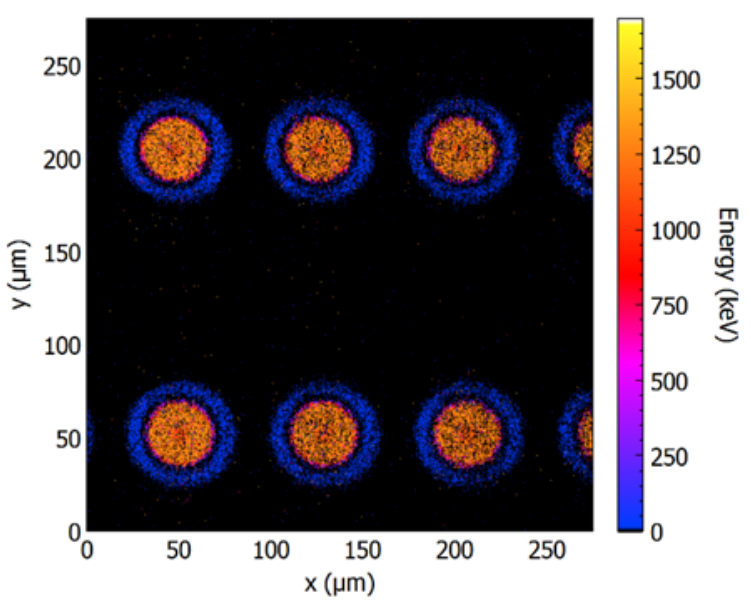

b)
Fig. 8 Response of even array of the trenched planar mushroom microdosimeter to $5.5 \mathrm{MeV} \mathrm{He}^{2+}$ a) Energy spectrum and b) median energy map for $10 \mathrm{~V}$ bias.
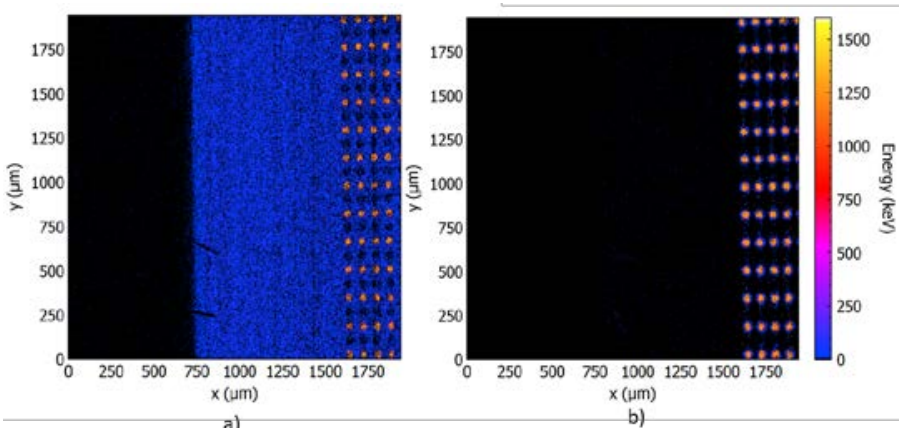

Fig. 9 Comparison of the median energy maps obtained near the edge of the planar trenched devices at a) $0 \mathrm{~V}$ bias and b) $5 \mathrm{~V}$ bias

Fig. 9 shows that at $0 \mathrm{~V}$ the low energy events were collected everywhere near the edge of the devices and between the SVs where the p-stop layer was deposited. When detector is biased at $5 \mathrm{~V}$, low energy events disappeared which is confirming that the p-stop layer is working when the detector is biased.
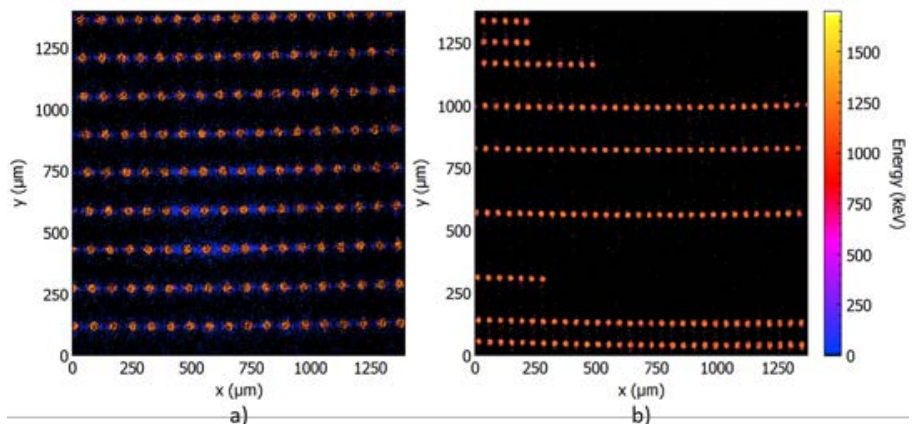

Fig. 10 Median energy map showing yield of trenched 3D (a) and trenched planar device structures (b), biased at $10 \mathrm{~V}$.

Fig. 10 shows that the trenched 3D device (trench filled with air) has $100 \%$ yield while the trenched planar device (trench filled with polysilicon) has many disconnected sensitive volumes. This is due to the severe topography of the surface which has polysilicon present and results in the breakage of the aluminium contacts over the filled trenches (see Fig. 3b).

\section{Determination of dose equivalent out of primary carbon} ion therapy field

The dose equivalent per Gy in the middle of the SOBP was calculated based on measured lineal energy spectra at 150, 160,180 and $240 \mathrm{~mm}$ depth in water. The range of $290 \mathrm{MeV} / \mathrm{u}$ ${ }^{12} \mathrm{C}$ ions in water is approximately $148 \mathrm{~mm}$. Fig. 11 shows that the dose equivalent just beyond the distal edge of the SOBP was $137 \mathrm{mSv} / \mathrm{Gy}$. It is due to contribution of some straggling carbon ions and heavy fragments such as boron $(\mathrm{Z}=5)$ and beryllium $(Z=4)$ which have slightly longer ranges than the carbon primaries created through nuclear interactions. Lighter fragmentation products such as $\mathrm{Li}$, He and $\mathrm{H}$ are dominating at a few $\mathrm{cm}$ beyond the SOBP. At $10 \mathrm{~cm}$ past the distal edge of the SOBP, the dose equivalent was $13 \mathrm{mSv} / \mathrm{Gy}$ and is mostly due to light fragments and fast neutrons. 


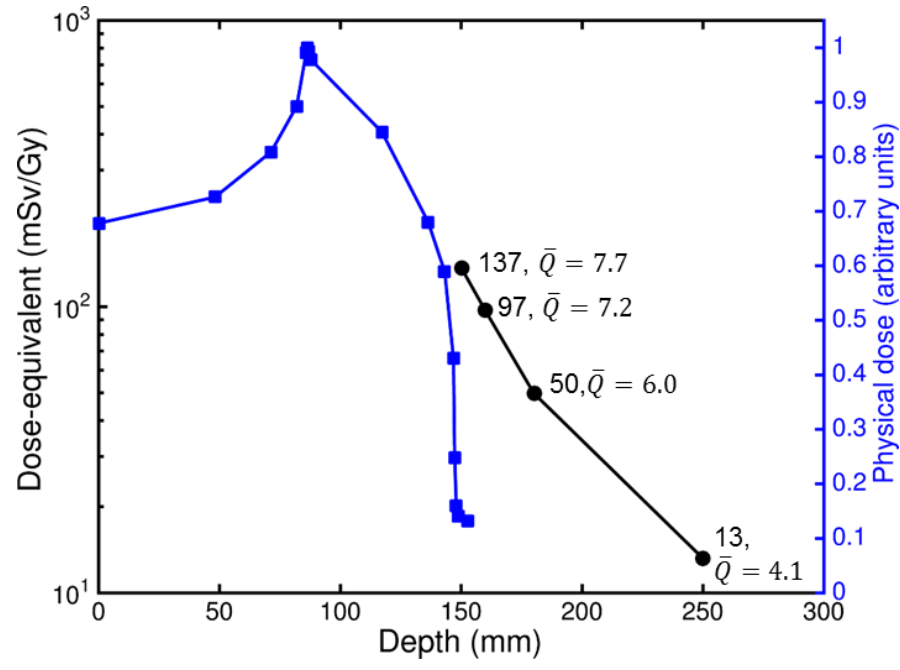

Fig. 11 Physical dose distribution along $60 \mathrm{~mm}$ SOBP delivered with the 290 $\mathrm{MeV} / \mathrm{u}{ }^{12} \mathrm{C}$ ion beam and dose equivalent downstream of the SOBP. An average Quality coefficient was calculated at each measurement point.

\section{CONCLUSION}

The silicon microdosimeters with 3D structures were fabricated and investigated in detail using the IBICC technique with $5.5 \mathrm{MeV} \mathrm{He}^{2+}$ microbeams. Well-defined SV shapes was observed in both of the 3D microdosimeter structures, however some very low energy events that exist due to the small bridge region of silicon in the trenched 3D structure and outer polysilicon filler in the trenched planar structure were observed. The planar $\mathrm{n}^{+}$core structure was found suitable for uniformity of charge collection within the 3D SV. TCAD simulations were performed to understand the electrical properties of the trenched 3D devices. 100\% yield was observed with the trenched 3D structures while lower yield has been observed with the trenched planar devices due to breakage of the aluminum contacts.

Application of the 3D mushroom microdosimeter in a heavy ion therapy radiation field demonstrated that these microdosimeters can be used for quality factor and dose equivalent measurements in mixed radiation fields similar to deep space radiation environment inside of spacecraft.

In the next version of the mushroom microdosimeter (trenched planar structure), the metal lines will be improved by increasing the pad area covering polysilicon trenches. The mushroom microdosimeter will be covered with PMMA to increase tissue equivalence when considering secondary particle production.

\section{ACKNOWLEDGEMENTS}

This research was supported by the Australian Government through the Australian Research Council's Discovery Projects funding scheme (project DP 170102273).

The authors wish to acknowledge the National Collaborative Research Infrastructure Strategy (NCRIS) funding provided by the Australian Government for this research.
The authors would like to acknowledge Dr. Nadia Court and her team at the UNSW ANFF node for their packaging work as well as Prof. Elena Pereloma and her electron microscopy team at the Australian Institute for Innovative Materials, University of Wollongong for their excellent imaging work. Finally the authors would like to thank all collaborators in 3D-MiMiC project, funded by the Norwegian Research Council.

\section{REFERENCES}

[1] International Commission on Radiation Units and Measurements, "The quality factor in radiation protection,” Report ICRU-40, 1986.

[2] H. H. Rossi and M. Zaider, "Microdosimetry and Its Applications," Springer, London, 1996.

[3] P. D. Bradley, "The development of a novel silicon microdosimeter for High LET radiation therapy,” Ph.D. dissertation, Fac. Eng., Univ. Wollongong, Wollongong, Australia, 2000.

[4] A. L. Ziebell, W. H. Lim, M. I. Reinhard, I. Cornelius, D. A. Prokopovich, R. Siegele, A. S. Dzurak, and A. B. Rosenfeld, “A cylindrical silicon-on-insulator microdosimeter: charge collection characteristics," IEEE Trans. Nucl. Sci., vol. 55, no. 6, pp. 3414-3420, Dec. 2008.

[5] D. A. Prokopovich, M. I. Reinhard, G. C. Taylor, A. Hands, A. B. Rosenfeld, "Comparison of SOI microdosimeter and tissue equivalent proportional counter measurements at the CERF facility," IEEE Trans. Nucl. Sci., vol. 59, no. 5, pp. 2501-2505, 2012.

[6] J. Livingstone, D. A. Prokopovich, M. L. F. Lerch, M. Petasecca, M. I. Reinhard, H. Yasuda, M. Zaider, J. Ziegler, V. L. Pisacane, J. Dicello, V. Perevetaylo, and A. B. Rosenfeld, "Large area silicon microdosimeter for dosimetry in high LET space radiation fields: charge collection study," IEEE Trans. Nucl. Sci., vol. 59, no. 6, pp. 3126-3132, Dec. 2012.

[7] L. T. Tran, Lachlan Chartier, Dale A. Prokopovich, Marco Petasecca, Michael L. F. Lerch, Mark I. Reinhard, Vladimir Perevetaylor, Naruhiro Matsufuji and Anatoly B. Rosenfeld, "3D-mesa "Bridge" silicon microdosimeter: charge collection study and application to RBE studies in ${ }^{12} \mathrm{C}$ radiation therapy," IEEE Trans. Nucl. Sci., vol. 62, no. 2, pp. 504511, Apr. 2015.

[8] L. T. Tran, Lachlan Chartier, David Bolst, Susanna Guatelli, Mark I. Reinhard, Marco Petasecca, Michael L. F. Lerch, Vladimir L. Pereverlaylo, Naruhiro Matsufuji, David Hinde, Mahananda Dasgupta, Andrew Stuchbery and Anatoly B. Rosenfeld, "3D Silicon Microdosimetry and RBE study using ${ }^{12} \mathrm{C}$ ion of different energies," IEEE Trans Nucl. Sci., vol. 62, no. 6, pp. 3027 - 3033, 2015.

[9] A. B. Rosenfeld, "Novel detectors for silicon based microdosimetry, their concepts and applications," Nucl. Instrum. Meth., Phys. Res. A, vol. 809, pp. 156-170, February 2016.

[10] I. Parker, C. J. Kenney, and J. Segal, "3D- A proposed new architecture for solid-state radiation detectors,” Nucl. Instrum. Meth., Phys. Res. A, vol. A395, pp. 328-343, 1997.

[11] L. T. Tran, Dale A. Prokopovich, Marco Petasecca, Michael L. F. Lerch, Angela Kok, Anand Summanwar, Cinzia Da Via, Mark I. Reinhard, Kari Schjlberg-Henriksenand, Anatoly B. Rosenfeld, "3D radiation detectors: charge collection characterisation and applicability of technology for microdosimetry,” IEEE Trans. Nucl. Sci., vol. 61, no. 4, pp. 1537-1543, Mar. 2014.

[12] L. T. Tran, Dale A.Prokopovich, Marco Petasecca, Michael L. F. Lerch, Celeste Fleta, Giulio Pellegrini, Consuelo Guardiola, Mark I. Reinhard and Anatoly B. Rosenfeld, "Ultra-thin 3D Detectors: Charge Collection Characterisation and Application for Microdosimetry,” IEEE Trans. Nucl. Sci., vol. 61, no. 6, pp. 3472-3478, 2014.

[13] R. Siegele, D. Cohen, and N. Dytlewski, "The ANSTO high energy heavy ion microprobe,” Nucl. Instrum. Meth. Phys. Res. B, vol. 158, pp. 31-38, 1999.

[14] Y. Kase, Y. Matsumoto, Y. Furusawa, H. Okamoto, T. Asaba, M. Sakama, and H. Shinoda, "Microdosimetric measurements and estimation of human cell survival for heavy-ion beams," Radiat. Res., vol. 166, no. 4, pp. 629-638, Oct. 2006.

[15] D. Bolst, S. Guatelli, L. T. Tran, L. Chartier, M. Lerch, N. Matsufuji and A. Rosenfeld, "Correction factors to convert microdosimetry measurements in silicon to tissue in ${ }^{12} \mathrm{C}$ ion therapy," Phys. Med. Biol., vol. 62, no. 6, pp. 2055-2069, 2017. 